\title{
Estimation of Unknown Function of a Class of Nonlinear Weakly Singular Integral Inequality
}

\author{
Chunmiao Huang and Wusheng Wang* \\ School of Mathematics and Statistics, Hechi University, Guangxi, Yizhou 546300, P. R. China \\ ${ }^{*}$ Corresponding author
}

\begin{abstract}
In this paper, we investigate a nonlinear weakly singular integral inequality. The upper bounds of the embedded unknown functions are estimated explicitly by the definitions and rules of conformable fractional differential and conformable fractional integration, the techniques of change of variable, and the method of amplification. The derived results can be applied in the study of qualitative properties of solutions of conformable fractional integral equations.
\end{abstract}

Keywords-weakly singular integral inequalities; conformable fractional integral; conformable fractional differential; analysis technique; explicit bound

\section{INTRODUCTION}

It is well known that integral equations are important tools to investigate the rule of natural phenomena. In the study of the qualitative properties of solutions of integral equations, one often deals with certain weakly singular integral inequalities. In 2008, Ma and Pecaric[1] investigated weakly singular integral inequality

$$
u^{p}(t) \leq a(t)+b(t) \int_{0}^{t}\left(t^{\alpha}-s^{\alpha}\right)^{\beta-1} s^{\gamma-1} f(s) u^{q}(s) d s .
$$

In 2014, Zheng [2] discussed the weakly singular integral inequalities of the following form

$$
\begin{gathered}
u(t) \leq C+\frac{1}{\Gamma(\alpha)} \int_{0}^{t}(t-s)^{\alpha-1} g(s) u(s) d s \\
+\frac{1}{\Gamma(\alpha)} \int_{0}^{A}(A-s)^{\alpha-1} g(s) u(s) d s . \\
u(t) \leq C+\int_{0}^{t} h(s) u^{p}(s) d s \\
+\frac{1}{\Gamma(\alpha)} \int_{0}^{t}(t-s)^{\alpha-1} g(s) u^{q}(s) d s
\end{gathered}
$$

$$
\begin{array}{r}
+\int_{0}^{A} h(s) u^{p}(s) d s \\
+\frac{1}{\Gamma(\alpha)} \int_{0}^{A}(A-s)^{\alpha-1} g(s) u^{q}(s) d s .
\end{array}
$$

With the development of the theory of differential equations, integral inequalities have been paid much attention by many authors. We refer to the papers [3-10] and the references cited therein.

In this paper, on the basis of [2, 7, 8], we discuss weakly singular integral inequality

$$
\begin{aligned}
& u(t) \leq c+\int_{a}^{t}(s-a)^{\alpha-1} f(s) w_{1}(u(s)) d s \\
& +\int_{a}^{t}(s-a)^{\alpha-1} g(s) w_{1}(u(s))[u(s) \\
& \left.+\int_{a}^{s}(\tau-a)^{\alpha-1} h(\tau) w_{2}(u(\tau)) d \tau\right] d s
\end{aligned}
$$

In order to investigate the integral inequality (4), we shall state some basic notations and lemmas, which will be used in the proofs of our main results.

Definition 1. (see [11, 12]) The (left) conformable fractional derivative starting from $a$ of a function $f:[a, \infty) \rightarrow R_{+}$of order $0<\alpha \leq 1$ is defined by

$$
T_{\alpha}^{a}(f)(t)=\lim _{\varepsilon \rightarrow 0} \frac{f\left(t+\varepsilon(t-a)^{1-\alpha}\right)-f(t)}{\varepsilon},
$$

for all $t>0$. If $f$ is $\alpha$-differentiable in some $[a, \infty)$, and $\lim _{t \rightarrow a^{+}} f^{(\alpha)}(t)$ exists, then define

$$
f^{(\alpha)}(0)=\lim _{t \rightarrow a^{+}} f^{(\alpha)}(t)
$$

Definition 2. (see $[11,12]$ ) The (left) conformable fractional integral starting from $a$ of a function $f:[a, \infty) \rightarrow R_{+}$of order $0<\alpha \leq 1$ is defined by 


$$
I_{\alpha}^{a}(f)(t)=\int_{a}^{t}(s-a)^{\alpha-1} f(s) d s
$$

Lemma 1. (see [11, 12]) Let $a, b, p, \lambda, \alpha$ are real constants with $\alpha \in(0,1]$ and $f, g$ be $\alpha$-differentiable at a point $t>0$.Let $h(t)=f(g(t))$.Then

$$
\begin{gathered}
T_{\alpha}^{a}(\lambda)=0, \\
T_{\alpha}^{a}\left(t^{p}\right)=p t^{p-\alpha}, \\
T_{\alpha}^{a}(a f+b g)=a T_{\alpha}^{a}(f)+b T_{\alpha}^{a}(g), \\
T_{\alpha}^{a}(f g)=f T_{\alpha}^{a}(g)+g T_{\alpha}^{a}(f), \\
T_{\alpha}^{a}\left(I_{\alpha}^{a}(f)\right)(t)=f(t), \\
I_{\alpha}^{a}\left(T_{\alpha}^{a}(f)\right)(t)=f(t)-f(a), \\
T_{\alpha}^{a}(h)(t)=T_{\alpha}^{a}(f)(g(t)) T_{\alpha}^{a}(g)(t) g^{\alpha-1}(t) .
\end{gathered}
$$

If, in addition, $f$ is differentiable, then

$$
T_{\alpha}^{a}(f)(t)=(t-a)^{1-\alpha} \frac{d f(t)}{d t} .
$$

\section{MAIN RESULT}

Throughout this paper, let $R_{+}=[0,+\infty)$. Define three functions by $w_{1}, w_{2}$ in (4)

$$
\begin{gathered}
W_{1}(t)=\int_{0}^{t} \frac{(s-a)^{\alpha-1} s^{1-\alpha} d s}{W_{1}(s)}, t \in R_{+} \\
W_{2}(t)=\int_{0}^{t} \frac{(s-a)^{\alpha-1} s^{1-\alpha} d s}{W_{1}^{-1}(s)}, t \in R_{+},
\end{gathered}
$$

$W_{3}(t)=\int_{0}^{t} \frac{(s-a)^{\alpha-1} W_{1}\left(W_{1}^{-1}\left(W_{2}^{-1}(s)\right)\right) W_{1}^{-1}\left(W_{2}^{-1}(s)\right) d s}{s^{\alpha-1} W_{2}\left(W_{1}^{-1}\left(W_{2}^{-1}(s)\right)\right)}$,

for all $t>0$.

Theorem

1. Suppose

that $f, g, h \in C\left(R_{+}, R_{+}\right), w_{1}, w_{2}, w_{2} / w_{1} \in C\left(R_{+}, R_{+}\right)$ar e all nondecreasing and positive functions, and $C$ is a nonnegative constant. If $u(t)$ satisfies (4), then

$$
u(t) \leq W_{1}^{-1}\left\{W_{2}^{-1}\left[W_{3}^{-1}(\Xi(t)]\right\}, t \in\left[0, T_{1}\right],\right.
$$

where

$$
\begin{gathered}
\Xi(t)=W_{3}\left\{W_{2}\left[W_{1}(c)+\int_{a}^{t}(s-a)^{\alpha-1} f(s) d s\right]\right. \\
\left.+\int_{a}^{t}(s-a)^{\alpha-1} g(s) d s\right\}+\int_{a}^{t}(s-a)^{\alpha-1} h(s) d s,
\end{gathered}
$$

and $T_{1}$ is the largest number such that

$$
\begin{aligned}
& \Xi(t) \leq W_{3}(\infty), W_{3}^{-1}\left(\Xi\left(T_{1}\right)\right) \leq W_{2}(\infty), \\
& W_{2}^{-1}\left(W_{3}^{-1}\left(\Xi\left(T_{1}\right)\right)\right) \leq W_{1}(\infty) .
\end{aligned}
$$

Proof. Define a function $z_{1}(t)$ by the right hand side of the inequality (4), i. e.

$$
\begin{array}{r}
z_{1}(t)=c+\int_{a}^{t}(s-a)^{\alpha-1} f(s) w_{1}(u(s)) d s \\
+\int_{a}^{t}(s-a)^{\alpha-1} g(s) w_{1}(u(s))[u(s) \\
\left.+\frac{1}{\Gamma(\alpha)} \int_{a}^{s}(\tau-a)^{\alpha-1} h(\tau) w_{2}(u(\tau)) d \tau\right] d s .
\end{array}
$$

We observe that $Z_{1}(t)$ is a positive and nondecreasing function on $[a, \infty)$. From (4) and (20) we have

$$
\begin{gathered}
u(t) \leq z_{1}(t), t \in[a, \infty), \\
z_{1}(a)=c .
\end{gathered}
$$

Using the define 2 of the (left) conformable fractional integral starting from $a$ of a function and (20) 


$$
\begin{gathered}
z_{1}(t)=c+I_{\alpha}^{a}\left(f(t) w_{1}(u(t))\right)+I_{\alpha}^{a}\left\{g(t) w_{1}(u(t))\right. \\
\left.\times\left[u(t)+\int_{a}^{t}(\tau-a)^{\alpha-1} h(\tau) w_{2}(u(\tau)) d \tau\right]\right\}, t \in R_{+} .
\end{gathered}
$$

By the lemma 1, we get

$$
\begin{gathered}
T_{\alpha}^{a}\left(z_{1}\right)(t)=f(t) w_{1}(u(t))+g(t) w_{1}(u(t))[u(t) \\
\left.+\int_{a}^{t}(\tau-a)^{\alpha-1} h(\tau) w_{2}(u(\tau)) d \tau\right], t \in R_{+} .
\end{gathered}
$$

From (21) and (24), we have

$$
\begin{aligned}
& T_{\alpha}^{a}\left(z_{1}\right)(t) \leq f(t) w_{1}\left(z_{1}(t)\right)+g(t) w_{1}\left(z_{1}(t)\right)\left[z_{1}(t)\right. \\
& \left.\left.+\int_{a}^{t}(\tau-a)^{\alpha-1} h(\tau) w_{2}\left(z_{1}(\tau)\right) d \tau\right]\right\}, t \in R_{+} .
\end{aligned}
$$

Define a function $z_{2}(t)$ by

$$
\begin{gathered}
z_{2}(t)=z_{1}(t)+\int_{a}^{t}(\tau-a)^{\alpha-1} h(\tau) w_{2}\left(z_{1}(\tau)\right) d \tau \\
=z_{1}(t)+I_{\alpha}^{a}\left(h(t) w_{2}\left(z_{1}(t)\right)\right), t \in R_{+} .
\end{gathered}
$$

Obviously, $z_{2}(t)$ is a positive and nondecreasing function on $[a, \infty)$. From (22) and (26), we have

$$
\begin{gathered}
z_{1}(t) \leq z_{2}(t), t \in[a, \infty), \\
z_{2}(a)=c .
\end{gathered}
$$

Making a conformable fractional derivative starting from $a$ of the function $z_{2}(t)$ of order $\alpha$, by the lemma 1 , we obtain

$$
\begin{aligned}
& T_{\alpha}^{a}\left(z_{2}\right)(t)=T_{\alpha}^{a}\left(z_{1}\right)(t)+h(t) w_{2}\left(z_{1}(t)\right) \\
& \leq f(t) w_{1}\left(z_{1}(t)\right)+g(t) w_{1}\left(z_{1}(t)\right) z_{2}(t)+h(t) w_{2}\left(z_{1}(t)\right) \\
& \leq f(t) w_{1}\left(z_{2}(t)\right)+g(t) w_{1}\left(z_{2}(t)\right) z_{2}(t) \\
& \quad+h(t) w_{2}\left(z_{2}(t)\right), t \in R_{+} .
\end{aligned}
$$

By the definition of $W_{1}$ and the lemma 1, from (29) we obtain

$$
T_{\alpha}^{a} W_{1}\left(z_{2}(t)\right)=\frac{z_{2}^{1-\alpha}(t)}{W_{1}\left(z_{2}(t)\right)} T_{\alpha}^{a}\left(z_{2}\right)(t) z_{2}^{\alpha-1}(t)
$$

$$
\leq f(t)+g(t) z_{2}(t)+h(t) \frac{w_{2}\left(z_{2}(t)\right)}{w_{1}\left(z_{2}(t)\right)}, t \in R_{+} .
$$

Substituting $t$ with $\tau$ in (30), making a conformable fractional integral of order $\alpha$ for (30) with respect to $\tau$ from 0 to $t$ and using the lemma 1 , we obtain

$$
\begin{gathered}
W_{1}\left(z_{2}(t)\right) \leq W_{1}\left(z_{2}(a)\right)+\int_{a}^{t}(s-a)^{\alpha-1} f(s) d s \\
+\int_{a}^{t}(s-a)^{\alpha-1} g(s) z_{2}(s) d s \\
+\int_{a}^{t}(s-a)^{\alpha-1} h(s) \frac{W_{2}\left(z_{2}(s)\right)}{W_{1}\left(z_{2}(s)\right)} d s \\
\leq W_{1}\left(z_{2}(a)\right)+\int_{a}^{T}(s-a)^{\alpha-1} f(s) d s \\
+\int_{a}^{t}(s-a)^{\alpha-1} g(s) z_{2}(s) d s
\end{gathered}
$$

$$
+\int_{a}^{t}(s-a)^{\alpha-1} h(s) \frac{w_{2}\left(z_{2}(s)\right)}{w_{1}\left(z_{2}(s)\right)} d s, t \in[a, T] .
$$

where $T \in\left[0, T_{1}\right]$ is chosen arbitrarily. Let $Z_{3}$ denote the right hand side of the inequality (31), i. e.

$$
\begin{array}{r}
z_{3}(t)=W_{1}\left(z_{2}(a)\right)+\int_{a}^{T}(s-a)^{\alpha-1} f(s) d s \\
+\int_{a}^{t}(s-a)^{\alpha-1} g(s) z_{2}(s) d s \\
+\int_{a}^{t}(s-a)^{\alpha-1} h(s) \frac{w_{2}\left(z_{2}(s)\right)}{w_{1}\left(z_{2}(s)\right)} d s, t \in[a, T] .
\end{array}
$$

which is a positive and nondecreasing function on $[a, T]$. From (31) and (32) we have

$$
z_{2}(t) \leq W_{1}^{-1}\left(z_{3}(t)\right), t \in[a, T]
$$

$$
z_{3}(a)=W_{1}\left(z_{2}(a)\right)+\int_{a}^{T}(s-a)^{\alpha-1} f(s) d s .
$$

From (32) and (33), we have

$$
\begin{array}{r}
\quad T_{\alpha}^{a}\left(z_{3}\right)(t)=g(t) z_{2}(t)+h(t) \frac{w_{2}\left(z_{2}(t)\right)}{w_{1}\left(z_{2}(t)\right)} \\
\leq g(t) W_{1}^{-1}\left(z_{3}(t)\right)+h(t) \frac{W_{2}\left(W_{1}^{-1}\left(z_{3}(t)\right)\right)}{w_{1}\left(W_{1}^{-1}\left(z_{3}(t)\right)\right)},
\end{array}
$$


for all $t \in[a, T]$.

Using the definition of $W_{2}$ and the lemma 1, from (35) we get

$$
\begin{gathered}
T_{\alpha}^{a}\left(W_{2}\left(z_{3}(t)\right)\right)=\frac{z_{3}^{1-\alpha}(t)}{W_{1}^{-1}\left(z_{3}(t)\right)} T_{\alpha}^{a}\left(z_{3}\right)(t) z_{3}^{\alpha-1}(t) \\
\leq g(t)+h(t) \frac{W_{2}\left(W_{1}^{-1}\left(z_{3}(t)\right)\right)}{w_{1}\left(W_{1}^{-1}\left(z_{3}(t)\right)\right) W_{1}^{-1}\left(z_{3}(t)\right)}, t \in[a, T] .
\end{gathered}
$$

Substituting $t$ with $\tau$ in (36), making a fractional integral of order $\alpha$ for (36) with respect to $\tau$ from 0 to $t$ and using the lemma 1 , we obtain that

$$
\begin{gathered}
W_{2}\left(z_{3}(t)\right) \leq W_{2}\left(z_{3}(a)\right)+\int_{a}^{t}(s-a)^{\alpha-1} g(s) d s \\
+\int_{a}^{t}(s-a)^{\alpha-1} h(s) \frac{W_{2}\left(W_{1}^{-1}\left(z_{3}(s)\right)\right)}{W_{1}\left(W_{1}^{-1}\left(z_{3}(s)\right)\right) W_{1}^{-1}\left(z_{3}(s)\right)} d s \\
\leq W_{2}\left(z_{3}(a)\right)+\int_{a}^{T}(s-a)^{\alpha-1} g(s) d s \\
+\int_{a}^{t}(s-a)^{\alpha-1} h(s) \frac{W_{2}\left(W_{1}^{-1}\left(z_{3}(s)\right)\right)}{W_{1}\left(W_{1}^{-1}\left(z_{3}(s)\right)\right) W_{1}^{-1}\left(z_{3}(s)\right)} d s
\end{gathered}
$$

for all $t \in[a, T]$.

Let $Z_{4}$ denote the right hand side of the inequality (37), i. e.

$$
\begin{gathered}
z_{4}(t)=W_{2}\left(z_{3}(a)\right)+\int_{a}^{T}(s-a)^{\alpha-1} g(s) d s \\
+\int_{a}^{t}(s-a)^{\alpha-1} h(s) \frac{W_{2}\left(W_{1}^{-1}\left(z_{3}(s)\right)\right)}{W_{1}\left(W_{1}^{-1}\left(z_{3}(s)\right)\right) W_{1}^{-1}\left(z_{3}(s)\right)} d s
\end{gathered}
$$

for all $t \in[a, T]$.

From (37) and (38) we have

$$
\begin{gathered}
Z_{3}(t) \leq W_{2}^{-1}\left(z_{4}(t)\right), t \in[0, T], \\
Z_{4}(a)=W_{2}\left(z_{3}(a)\right)+\int_{a}^{T}(s-a)^{\alpha-1} g(s) d s .
\end{gathered}
$$

From (38) and (39) we have

$$
T_{\alpha}^{a}\left(z_{4}\right)(t)=h(t) \frac{w_{2}\left(W_{1}^{-1}\left(z_{3}(t)\right)\right)}{w_{1}\left(W_{1}^{-1}\left(z_{3}(t)\right)\right) W_{1}^{-1}\left(z_{3}(t)\right)}
$$

$$
\leq h(t) \frac{w_{2}\left(W_{1}^{-1}\left(W_{2}^{-1}\left(z_{4}(t)\right)\right)\right)}{w_{1}\left(W_{1}^{-1}\left(W_{2}^{-1}\left(z_{4}(t)\right)\right)\right) W_{1}^{-1}\left(W_{2}^{-1}\left(z_{4}(t)\right)\right)},
$$

for all $t \in[0, T]$.

From (41) we get

$$
\begin{aligned}
& T_{\alpha}^{a}\left(W_{3}\left(z_{4}(t)\right)\right)=\frac{W_{1}\left(W_{1}^{-1}\left(W_{2}^{-1}\left(z_{4}(t)\right)\right)\right) W_{1}^{-1}\left(W_{2}^{-1}\left(z_{4}(t)\right)\right)}{z_{4}^{\alpha-1}(t) w_{2}\left(W_{1}^{-1}\left(W_{2}^{-1}\left(z_{4}(t)\right)\right)\right)} \\
& \quad \times T_{\alpha}^{a}\left(z_{4}(t)\right) z_{4}^{\alpha-1}(t) \leq h(t), t \in[a, T] .
\end{aligned}
$$

From (42) we have

$$
W_{3}\left(z_{4}(t)\right) \leq W_{3}\left(z_{4}(a)\right)+\int_{a}^{t}(s-a)^{\alpha-1} h(s) d s,
$$

for all $t \in[0, T]$.

From (21), (27), (33) and (39), we get

$$
\begin{gathered}
u(t) \leq z_{1}(t) \leq z_{2}(t) \leq W_{1}^{-1}\left(z_{3}(t)\right) \\
\leq W_{1}^{-1}\left(W_{2}^{-1}\left(z_{4}(t)\right)\right), t \in[a, T] .
\end{gathered}
$$

From (28), (34), (40), (43) and (44), we have

$$
\begin{gathered}
u(t) \leq W_{1}^{-1}\left\{W _ { 2 } ^ { - 1 } \left[W _ { 3 } ^ { - 1 } \left(W _ { 3 } \left(W _ { 2 } \left(W_{1}(c)\right.\right.\right.\right.\right. \\
\left.\left.+\int_{a}^{T}(s-a)^{\alpha-1} f(s) d s\right)+\int_{a}^{T}(s-a)^{\alpha-1} g(s) d s\right) \\
\left.\left.\left.+\int_{a}^{t}(s-a)^{\alpha-1} h(s) d s\right)\right]\right\}, t \in[0, T] .
\end{gathered}
$$

Because $T \in\left[0, T_{1}\right]$ is chosen arbitrarily, we obtain the required estimation (17). The proof is completed.

\section{SUMMARY}

In this paper, we investigate a nonlinear weakly singular integral inequality

$$
\begin{aligned}
u(t) & \leq c+\int_{a}^{t}(s-a)^{\alpha-1} f(s) w_{1}(u(s)) d s \\
& +\int_{a}^{t}(s-a)^{\alpha-1} g(s) w_{1}(u(s))[u(s) \\
& \left.+\int_{a}^{s}(\tau-a)^{\alpha-1} h(\tau) w_{2}(u(\tau)) d \tau\right] d s .
\end{aligned}
$$


By the definitions and rules of conformable fractional differential and conformable fractional integration, the techniques of change of variable and the method of amplification, we obtain the upper bounds of the embedded unknown functions:

$$
\begin{gathered}
u(t) \leq W_{1}^{-1}\left\{W _ { 2 } ^ { - 1 } \left[W _ { 3 } ^ { - 1 } \left(W _ { 3 } \left(W _ { 2 } \left(W_{1}(c)\right.\right.\right.\right.\right. \\
\left.\left.+\int_{a}^{T}(s-a)^{\alpha-1} f(s) d s\right)+\int_{a}^{T}(s-a)^{\alpha-1} g(s) d s\right) \\
\left.\left.\left.+\int_{a}^{t}(s-a)^{\alpha-1} h(s) d s\right)\right]\right\}, t \in[0, T] .
\end{gathered}
$$

\section{ACKNOWLEDGMENT}

This research was supported by National Natural Science Foundation of China( No. 11561019,11161018), Guangxi Natural Science Foundation (Project No. 2012GX NSFAA053009), Scientific Research Foundation of the Education Department of Guangxi Autonomous Region of China (No. KY2015ZD103, KY2015LX341), and the high school specialty and curriculum integration project of Guangxi Zhuang Autonomous Region (No. GXTSZY2220).

\section{REFERENCES}

[1] Q.H. Ma and J. Pecaric,“Some new explicit bounds for weakly singular integral inequalities with applications to fractional dierential and integral equations.” J. Math. Anal. Appl.341(2), PP.894-905, 2008.

[2] B. Zheng, "Explicit bounds derived by some new inequalities and applications in fractionalintegral equations," Journal of Inequalities and Applications, 2014(4), PP. 1-12, 2014.

[3] O. Lipovan,“ A retarded Gronwall-like inequality and its applications, ” J Math Anal Appl.,252, PP.389-401, 2000.

[4] B. G. Pachpatte, "Explicit bound on a retarded integral inequality,” Math Inequal Appl., 7, PP.7-11, 2004.

[5] R.P. Agarwal, S. Deng and W. Zhang, "Generalization of a retarded Gronwall-like inequalityand its applications,” Appl Math Comput., 165, PP.599-612, 2005.

[6] Q.H. Ma, J. Pecaric, "Estimates on solutions of some new nonlinear retarded Volterra-Fredholm type integral inequalities,” Nonlinear Anal., 69, PP.393-407, 2008.

[7] A. Abdeldaim and M. Yakout, "On some new integral inequalities of Gronwall-Bellman-Pachpatte type,” Appl Math Comput., 217, PP.78877899, 2011.

[8] H. El-Owaidy, A. Abdeldaim and A. A. El-Deeb, "On some new retarded nonlinear integral inequalities and theirs Applications," Mathematical Sciences Letters, 3(3), PP.157-164, 2014.

[9] G. C. Wu and E. W. M. Lee, "Fractional variational iteration method and its application,” Phys. Lett. A, 374, PP.2506-2509, 2010.

[10] B. Zheng, “( $\left.\mathrm{G}^{\prime} / \mathrm{G}\right)$-expansion method for solving fractional partial di erential equations in the theory of mathematical physics." Commun. Theor. Phys., 58, PP.623-630, 2012.

[11] R. Khalil, M. Al Horani, A. Yousef and M. Sababheh, “A new Denition Of Fractional Derivative,” J. Comput. Appl. Math. 264. pp. 6570, 2014.

[12] T. Abdeljawad, “On conformable fractional calculus,” J. Computational Appl. Math. 279, PP. 57-66, 2015 